\title{
Characterization, Thermal and Biological Properties of PCL/PLA/PEG/N-HA Composites
}

\author{
Mun Kit Fong ${ }^{1}$, Mohd Nasrull Abdol Rahman ${ }^{1}$, Ahmad Mubarak Tajul Arifin ${ }^{2}$, \\ Reazul Haq Abdul Haq 1,*iD, Mohd Fahrul Hassan ${ }^{2}$, Ishkrizat Taib ${ }^{2}$ \\ 1 Department of Manufacturing, Faculty of Mechanical and Manufacturing Engineering, Universiti Tun Hussien Onn, 86400 \\ Batu Pahat, Johor, Malaysia \\ 2 Department of Mechanical, Faculty of Mechanical and Manufacturing Engineering, Universiti Tun Hussien Onn, 86400 \\ Batu Pahat, Johor, Malaysia \\ * Correspondence: reazul@utm.edu.my;
}

Scopus Author ID 57211349523

Received: 17.07.2020; Revised: 23.08.2020; Accepted: 25.08.2020; Published: 30.08.2020

\begin{abstract}
Initially, the use of Additive Manufacturing (AM) technology is mainly for the production of prototypes of a new invention and design. However, the AM technology had extended to the tooling industry, medical scaffolding, and direct part production. This is due to the extensive research and development carried out around the globe regardless of the material, equipment, and technology of AM. In this study, characterization and properties of PCL/PLA/PEG/Nano-HA polymer composites were investigated. Polycaprolactone (PCL), polylactic acid (PLA), polyethylene glycol (PEG), and nanohydroxyapatite (n-HA) composites were prepared by melting and compounding the mixture using a Brabender machine. The composition of the materials was determined by previous research that it is the optimum recipe, with the ratio of PCL:PLA is 7:3 while the composition of PEG and nano-HA is 5\% per hundred resin each. The temperature of the Brabender machine was set at $160^{\circ} \mathrm{C}$, and the speed of the mixer was set at $30 \mathrm{rpm}$. After the mixing, the composites were characterized by using Fourier Transform Infrared Spectroscopy (FTIR) to determine the spectrum and quality of the composite mixed. Based on the spectrum, it can be verified that the characteristic of n-HA powder had been incorporated well into the PCL/PLA/PEG polymer blend. The thermal properties of the composites were investigated by using Thermogravimetry Analyzer (TGA). The results showed that the addition of n-HA lowered the initial degradation temperature and peak degradation temperature. Apparently, the addition of nHA did not increase the degradation temperature of the composites. Furthermore, Simulated Body Fluid (SBF) test was carried out to access the bioactivity properties of the composites after the addition of $\mathrm{n}$ HA. The results had proved that the addition of n-HA had triggered the growth of apatite layer on the surface of the samples treated with SBF. The growth of the apatite layer was verified by the X-ray Diffraction pattern, and the results proved the initial assumptions.
\end{abstract}

Keywords: Characterization; Thermal properties; Biological properties; PCL/PLA/PEG/n-HA; TGA; FTIR.

(C) 2020 by the authors. This article is an open-access article distributed under the terms and conditions of the Creative Commons Attribution (CC BY) license (https://creativecommons.org/licenses/by/4.0/).

\section{Introduction}

Additive Manufacturing (AM) is one of the manufacturing technology used to produce a solid physical model of an object through layer-by-layer fabrication technique of three dimensional (3D) structures that have been design from CAD software [1,2]. Fused Deposition Modelling (FDM) is a type of AM that build parts or prototypes with materials in the form of filament feedstock. It prints the products or parts by melting the filament, mostly thermoplastic 
filament, then extrudes and deposit layer by layer on the platform based on the design of STL file input. Among all the types of methods in 3D printing, the FDM 3D printer had become the most popular and affordable type [3, 4].

Polycaprolactone (PCL) and Polylactic Acid (PLA) are often used to fabricate medical implants as well as drug delivery applications. The degradation properties of PCL and PLA make them perfect for bone implants because they will gradually transfer the load from the support structure to the body as the bone heals. At the same time, PEG is normally used in laxative, a substance that is used to increase bowel movements. From previous research, the blends of PLA/PEG were better hydrolyzed by microorganisms than PLA/PCL blends, which means that the PLA/PEG blends degrade at a faster rate. The research had identified that the PEG had facilitated the biodegradation of PLA polymer. Hence, a proper amount of PEG had added to PCL/PLA blend to aid the degradation rate of PLA/PCL blends. With the addition of HA bio-ceramic into the PLA/PCL/PEG composite, how will the HA change the properties of the former composite [5].

Hydroxyapatite, also known as Hydroxyl-apatite (HA) is a natural mineral in the form of calcium apatite. It usually appears as white color. However, it also has a brown, yellow, or green color similar to the color of dental fluorosis. HA material is known for biological usage due to its material characteristics, which similar to human hard tissues [6]. The characteristics of good stability under physiological conditions of temperature, ph., and composition in human body fluid, making HA suitable to be further research of its usage in the human body. In human bone, $50 \%$ by volume and $70 \%$ by weight is a modified form of HA, known as a bone mineral. HA crystals are also found in the small calcifications of the pineal gland. Examples of ha in medical uses are bone grafting materials, dental prosthetics and repair parts, and some human body part implants. The incorporation of HA into current PCL/PLA/PEG composite is expected to enhance the properties of PCL/PLA/PEG composite to better suit into biological and medical usage purpose. The new PCL/PLA/PEG/HA composite is to be used for fused deposition modeling (FDM) as feedstock filament with the potential to be commercialized in the future.

The development of PCL/PLA/PEG/Nano-HA composite as a new biocompatible polymeric composite is believed to have huge potential for biomedical implants and bone tissue engineering applications. The newly developed material has the potential to be fully commercialized after all the related testing and studies were done provided if the result of the testing meets the OSHA standards and medical safety level. The addition of the Nano-HA material into PCL/PLA/PEG composite is expected to improve the bioactive and formation of bone-like apatite of the previous composites.

\section{Materials and Methods}

\subsection{Materials.}

The material used in this research was Polycaprolactone (PCL) medical grade BGH600C, Poly (lactic) acid (PLA) medical grade PTG600C, Polyethylene glycol (PEG) medical grade PEG 10000 and Nano-hydroxyapatite powder.

\subsection{Sample preparation.}

The materials were blended together by using a Brabender Plastograph EC. The blending temperature was set at $160^{\circ} \mathrm{C}$, with the rotating speed of $30 \mathrm{rpm}$. The composition of each material was summarized in Table 1. 
Table 1. Composition of materials.

\begin{tabular}{l|l} 
Material & Composition \\
\hline PCL & $70 \mathrm{wt} \%$ \\
\hline PLA & $30 \mathrm{wt} \%$ \\
\hline PEG & $5 \mathrm{phr}$ \\
\hline n-HA & $5 \mathrm{phr}$
\end{tabular}

The PCL/PLA was mixed first with a composition of 70 and $30 \mathrm{wt} \%$ respectively, the PEG and n-HA was added subsequently by $5 \%$ per hundred resin.

\subsection{Characterization by Fourier Transform Infrared (FTIR).}

FTIR is a spectrometer analysis that helps researchers to understand more of the materials and products. It is a technique used to obtain the infrared spectrum of absorption or emission of a solid, liquid, or gas, as shown in. The FTIR converts the detector output to the interpretable spectrum and generates spectra with a pattern that provides structural insights. When Infrared (IR) radiation passed through a sample, some of it was absorbed by the sample, and others passed through it, the simple layout inside the FTIR spectrometer is shown on. The resulting signal at the detector is a spectrum representing the 'fingerprint' of the sample. Since different materials have different chemical structures. Therefore each material will produce different spectral fingerprints.

The spectrum of PCL/PLA/PEG/Nano-HA composite was tested in the FTIR equipment, and the spectrum of the composite was captured and analyzed accordingly by the characteristic IR band positions.

\subsection{Thermal properties test.}

In this study, a TA TGA 550 Thermogravimetry Analyzer (TGA) apparatus was used to study the thermal stability of PCL/PLA/PEG/Nano-HA sample. Thermogravimetry analysis (TGA) is a technique to monitor the mass of a material or substance as a function of temperature or time as the sample is subjected to a controlled temperature environment. In a simple definition, TGA measures the sample's weight as the temperature increased or decreased.

The TGA software display a curve for the user to analyze the data, the X-axis of the TGA curve is usually displayed as time or temperature, and the Y-axis is usually displayed as weight (mg) or weight percent (wt\%).

\subsection{Biological test.}

In this study, SBF solution was prepared to conduct a bio-activity test (in vitro) for the PCL/PLA/PEG/Nano-HA sample. The SBF solution was made based on the recipe of Tadashi Kobuko [1]. The composition and standard operation procedure of the preparation of SBF solution are shown in Table 2. The comparison between human blood plasma and SBF fluid is demonstrated.

Table 2. Composition of preparing $1000 \mathrm{ml}$ of SBF [1].

\begin{tabular}{l|l|l|l|l|l} 
Order & Reagent & Amount & Container & Purity $(\%)$ & Formula weight \\
\hline $\mathbf{1}$ & $\mathrm{NaCl}$ & $8.035 \mathrm{~g}$ & Weighing paper & 99.5 & $\mathbf{5 8 . 4 4 3 0}$ \\
\hline $\mathbf{2}$ & $\mathrm{NaHCO} 3$ & $0.355 \mathrm{~g}$ & Weighing paper & 99.5 & $\mathbf{8 4 . 0 0 6 8}$ \\
\hline $\mathbf{3}$ & $\mathrm{KCl}$ & $0.225 \mathrm{~g}$ & Weighing paper & 99.5 & $\mathbf{7 4 . 5 5 1 5}$ \\
\hline $\mathbf{4}$ & $\mathrm{K} 2 \mathrm{HPO} 4 \cdot 3 \mathrm{H} 2 \mathrm{O}$ & $0.231 \mathrm{~g}$ & Weighing paper & 99.0 & $\mathbf{2 2 8 . 2 2 2 0}$ \\
\hline $\mathbf{5}$ & $\mathrm{MgCl} 2 \cdot 6 \mathrm{H} 2 \mathrm{O}$ & $0.311 \mathrm{~g}$ & Weighing paper & 98.0 & $\mathbf{2 0 3 . 3 0 3 4}$ \\
\hline $\mathbf{6}$ & $1.0 \mathrm{M} \mathrm{HCl}$ & $39 \mathrm{ml}$ & Weighing paper & - & - \\
\hline $\mathbf{7}$ & $\mathrm{CaCl} 2$ & $0.292 \mathrm{~g}$ & Weighing paper & 95.0 & $\mathbf{1 1 0 . 9 8 4 8}$
\end{tabular}




\begin{tabular}{l|l|l|l|l|l} 
Order & Reagent & Amount & Container & Purity $(\%)$ & Formula weight \\
\hline $\mathbf{8}$ & Na2SO4 & $0.072 \mathrm{~g}$ & Weighing paper & 99.0 & $\mathbf{1 4 2 . 0 4 2 8}$ \\
\hline $\mathbf{9}$ & Tris & $6.118 \mathrm{~g}$ & Weighing paper & 99.0 & $\mathbf{1 2 1 . 1 3 5 6}$ \\
\hline $\mathbf{1 0}$ & $\mathbf{1 . 0} \mathbf{M}$ HCl & $\mathbf{0 - 5} \mathbf{~ m l}$ & Weighing paper & - & -
\end{tabular}

The standard procedure of preparing 1000ml of SBF solution are as follow [1]:

- $700 \mathrm{ml}$ of ultra-pure water was poured into a $1000 \mathrm{ml}$ beaker and stirred using a magnetic bar at $36.5 \pm 0.2^{\circ} \mathrm{C}$.

- The reagents were dissolved in water in order numbers $1-8$, as in Table 2. Each reagent was completely dissolved before the next new reagent is added.

- The temperature of the solution was set at $36.5\left( \pm 1.5^{\circ} \mathrm{C}\right)$. Pure water was added up to 900 $\mathrm{mL}$.

- The electrode of the $\mathrm{pH}$ meter was inserted before dissolving Tris ( $9^{\text {th }}$ reagent $)$, and the $\mathrm{pH}$ level of the solution should be $2.0( \pm 1.0)$.

- A small amount of Tris was added until every reagent was dissolved completely, and the $\mathrm{pH}$ value becomes constant. After the $\mathrm{pH}$ value reached $7.30( \pm 0.05)$, the temperature is kept at $36.5\left( \pm 0.5^{\circ} \mathrm{C}\right)$. More Tris is added to increase the $\mathrm{pH}$ level to 7.45.

- $1 \mathrm{M} \mathrm{HCl}$ was dropped through a pipette to lower the $\mathrm{pH}$ level to approximately $7.42( \pm$ $0.01)$.

- The remaining Tris and $1 \mathrm{M} \mathrm{HCl}$ were poured alternately into the solution to maintain the $\mathrm{pH}$ level within the range of $7.42-7.45$.

- The $\mathrm{pH}$ level of the solution is then adjusted to 7.40 by dropping $1 \mathrm{M} \mathrm{HCl}$ dropwise at $36.5^{\circ} \mathrm{C}$.

- The solution was transferred to $1000 \mathrm{~mL}$ volumetric flask, and pure water was added up to the marked line after the temperature has cooled down to $20^{\circ} \mathrm{C}$.

\section{Results and Discussion}

\subsection{Fourier Transform Infrared.}

The FTIR spectra of PCL/PLA/PEG/n-HA is shown in Figure 1. The characteristic of both PCL/PLA/PEG polymer blend and n-HA are seen in the spectra, which are in agreement with the findings in [2] and [3]. From the spectra of PCL/PLA/PEG/n-HA, the main peaks occur at $1720 \mathrm{~cm}^{-1}\left(\mathrm{C}=\mathrm{O}\right.$ non-conjugated stretching), $1241 \mathrm{~cm}-1$, and $1160 \mathrm{~cm}^{-1}$ (C-O-C vibrations in Esters). The peaks at $1241 \mathrm{~cm}^{-1}$ and $1160 \mathrm{~cm}^{-1}$ are believed to be of Acetates and Formates, respectively. The characteristic of free $\mathrm{O}-\mathrm{H}$ bond and Intramolecular $\mathrm{H}$ bonds can be found in the region of $3610-3645 \mathrm{~cm}^{-1}$ (OH stretching vibrations) and $3450-3600 \mathrm{~cm}^{-1}$, respectively, with sharp peaks.

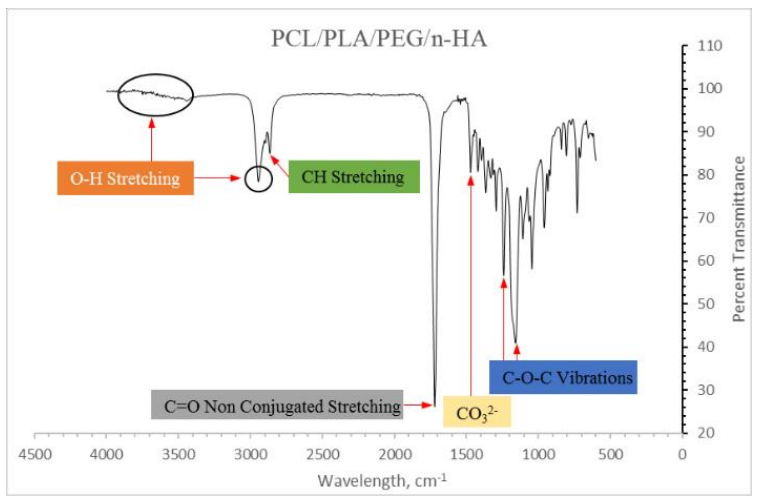

Figure 1. FTIR spectra of PCL/PLA/PEG/n-HA. 
The characteristic of n-HA can be found by the peak of OH- chemical group at 2945 $\mathrm{cm}-1$ and the intensive peak of CO 2- at $1472 \mathrm{~cm}-1$. Therefore, it can be concluded that the nHA had successfully incorporated into the PCL/PLA/PEG polymer blend.

\subsection{Thermogravimetry analysis (TGA).}

Thermogravimetric analysis was carried out to investigate the effect of blending composition on the thermal stability on the polymers. The weight loss in percent due to degradation of the sample at high temperature is monitored. The parameter of the TGA was set at an increment of $10{ }^{\circ} \mathrm{C} / \mathrm{min}$ from $0{ }^{\circ} \mathrm{C}$ ramp to $500{ }^{\circ} \mathrm{C}$, the balance gas used was Nitrogen gas at a flow rate of $25 \mathrm{~mL} / \mathrm{min}$. The use of inert gas flow allows the non-oxidative degradation to occur.

The TGA curves of PCL/PLA/PEG/n-HA composite are shown in Figure 2 and Figure 3. The initial thermal stability is evaluated by the temperature at $10 \%$ weight loss of the total weight of the sample tested, $\mathrm{T}_{10 \%}$. The peak temperature where the decomposition of the sample, Tp, happens also discovered from the data of the TGA curve.

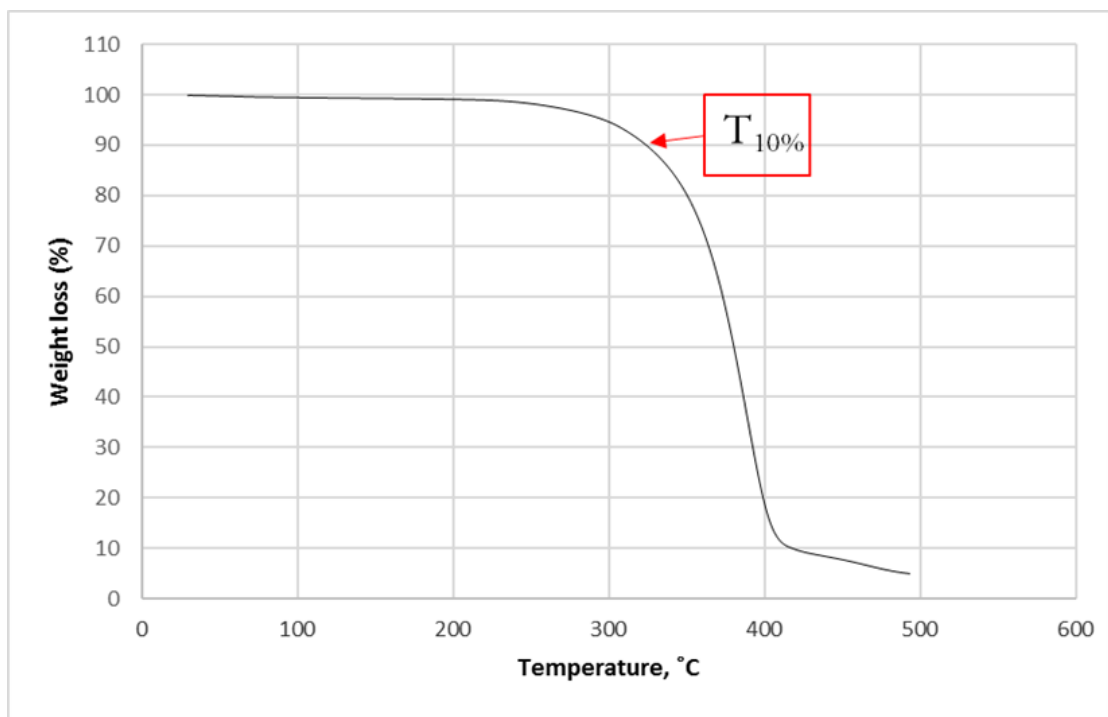

Figure 2. Thermogram of PCL/PLA/PEG/n-HA.

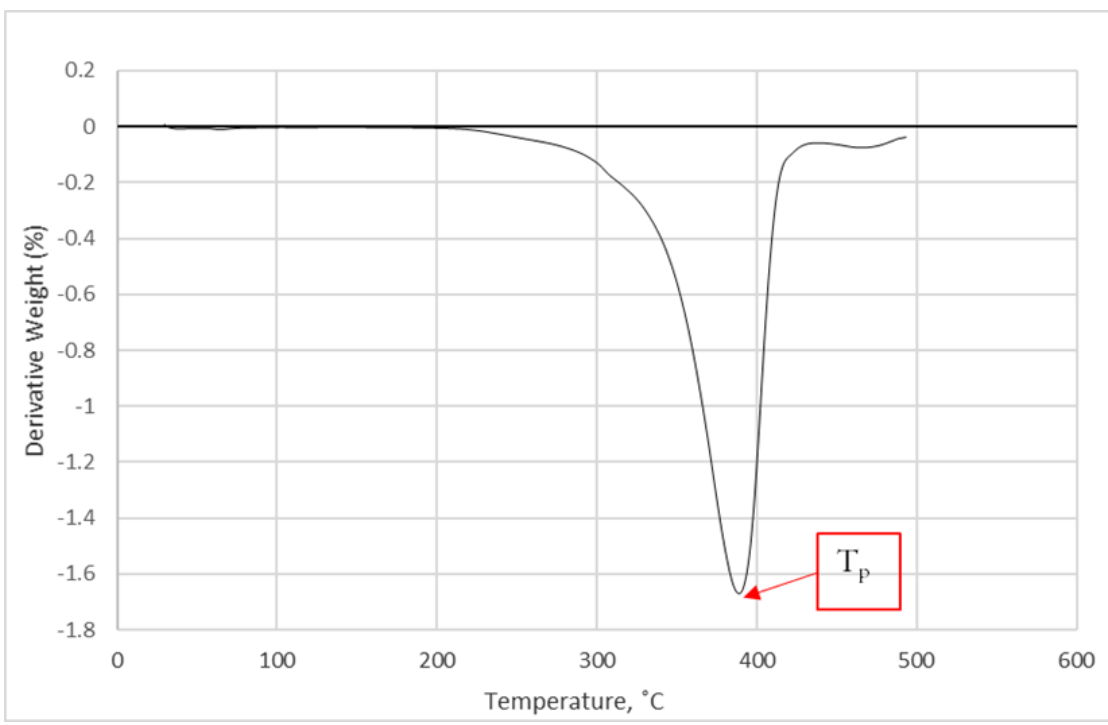

Figure 3. Derivatives thermogram of PCL/PLA/PEG/n-HA. 
Meanwhile, Table 3 concludes the $\mathrm{T}_{10 \%}, \mathrm{Tp}$, and weight of char residue of PCL/PLA/PEG/n-HA and PCL/PLA/PEG composites after thermogravimetric analysis.

Table 3. The initial decomposition, Peak decomposition, and weight of residue fo PCL/PLA/PEG/n-HA

\begin{tabular}{c|c|c|c|c} 
Designation & $\begin{array}{c}\text { Initial decomposition } \\
\left.\text { temperature, T10\% ( }{ }^{\circ} \mathbf{C}\right)\end{array}$ & $\begin{array}{c}\text { Peak of decomposition } \\
\left.\text { temperature, Tp ( }{ }^{\circ} \mathbf{C}\right)\end{array}$ & $\begin{array}{c}\text { Transition from } \\
\left.\text { T10\% to Tp, ( }{ }^{\circ} \mathbf{C}\right)\end{array}$ & $\begin{array}{c}\text { Weight of } \\
\text { residue (wt\%) }\end{array}$ \\
\hline PCL/PLA/PEG/n-HA & 323.7 & 389.4 & 65.7 & 4.97
\end{tabular}

Based on previous researches on n-HA enhanced polymer, the addition of hydroxyapatite had shown the potential to improve the thermal stability and resistance to thermal degradation of a polymer such as a polyamide 66 [4]. However, the initial decomposition temperature, $\mathrm{T} 10 \%$ and peak decomposition temperature, $\mathrm{Tp}$ of PCL/PLA/PEG/n-HA have decrease from $370.75^{\circ} \mathrm{C}$ to $323.7^{\circ} \mathrm{C}(\mathrm{T} 10 \%)$ and $400.1{ }^{\circ} \mathrm{C}$ to 389.4 ${ }^{\circ} \mathrm{C}$ (Tp) compared to the former material PCL/PLA/PEG composite. This proves that the incorporation of hydroxyapatite does not necessarily improve the thermal stability of polymer blends; it could also reduce the thermal stability of a polymer blend $[6,7]$.

From the graph of the thermogram of PCL/PLA/PEG/n-HA, there is a gap between the graph line and the origin axis. This represents the amount of char residue, $4.97 \mathrm{wt} \%$, resulting from the formation of a carbonaceous char layer from the burning of hydroxyapatite incorporated in the composite [8].

All in all, the conclusion that can be drawn from the results of TGA is that the thermal stability of PCL/PLA/PEG/n-HA is relatively stable by looking at the thermal degradation transition from $\mathrm{T} 10 \%$ to $\mathrm{Tp}$ where it takes $65.7{ }^{\circ} \mathrm{C}$ to reach the peak of thermal degradation from $\mathrm{T} 10 \%$ which is the double than that for PCL/PLA/PEG $\left(29.95{ }^{\circ} \mathrm{C}\right)$ polymer blend. However, the incorporation of HA does not necessarily improve the initial decomposition temperature, as proven by the initial decomposition temperature of PCL/PLA/PEG/n-HA $[9,10]$.

\subsection{Simulated body fluid test (SBF).}

In this analysis, the bioactivity of the PCL/PLA/PEG/n-HA composite is evaluated and assessed by growth or formation of carbonate apatite on the surface of the tested sample under simulated body fluid (SBF) test. The SEM microscope was used to observe and capture the changes to the sample's surface for immersion of samples in SBF for 3 days, 5 days, and 7 days.

The pattern of the apatite layer formed on the surface can differ in shape. The apatite layer form on a composite sample can be spherical crystal, leaf-like tree form or globular shape [11]. The formation of the apatite crystal layer on the sample surface was mainly due to the ion exchange reaction during immersion in the SBF. The newly formed layer caused the precipitation of $\mathrm{HA}$ by consuming $\mathrm{Ca}^{2+}$ and $\mathrm{PO}^{3+}$ ion from the SBF solution.

The surface morphology of treated samples of PCL/PLA/PEG/n-HA is shown in Figure 4. It can be seen from Figure 4 (a) where the immersion period is 3 days; there are some leaflike tree shapes (circled in red with labeled A) or pattern formed on the surface of the sample. This indicates that there is a possible growth of the apatite layer on the sample's surface after SBF test. After 5 days of immersion in SBF, the area of possible apatite layer formed was increase, and denser compared to 3 days of immersion. The shapes of the layer formed are in the shape of the globule, as shown in Figure 4 (b). 
Lastly, for the sample of 7 days immersion, the formation of the apatite layer is less noticeable compare to 3 days and 5 days. This may probably be due to the denser growth of the apatite layer or thickening of the apatite layer formed on the surface. Based on Figure 4 (c), it can be observed that the pores in the sample are smoother and covered up by the growth of the thin layer where it is believed to be due to the presence of the apatite layer [18].

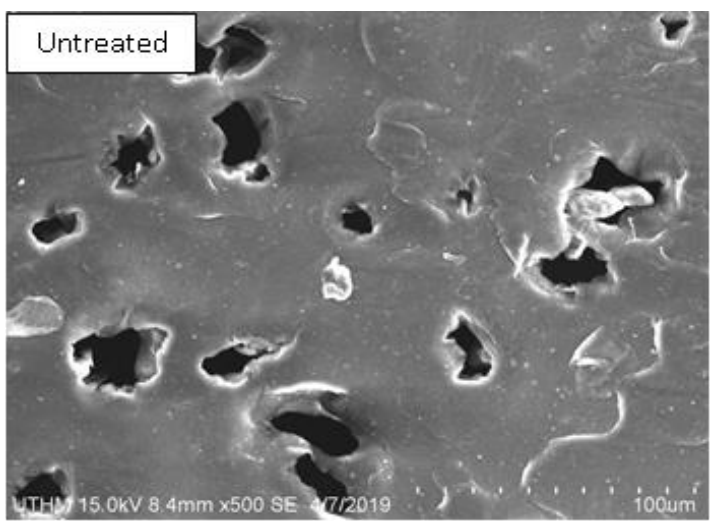

(a)

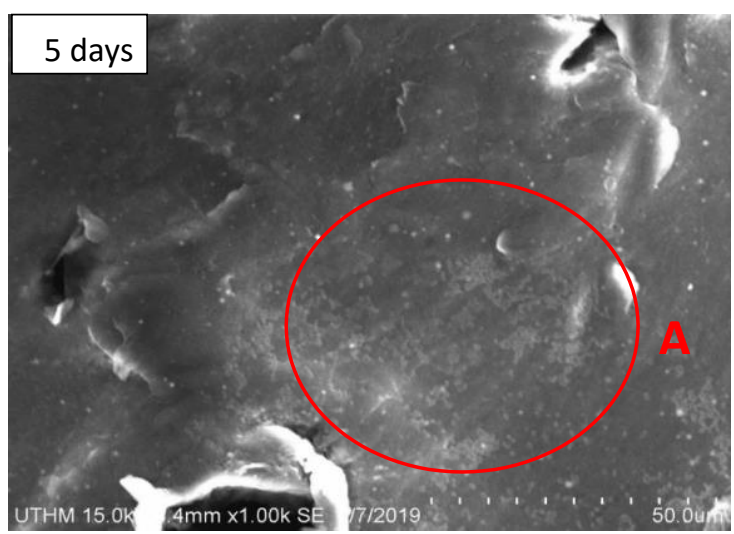

(c)

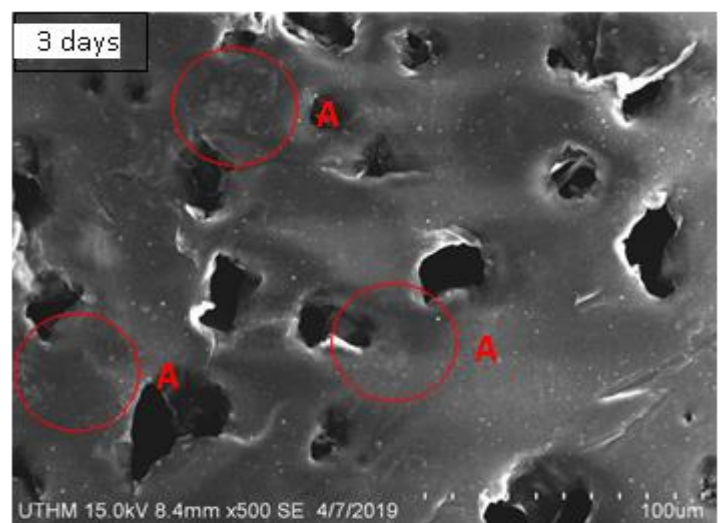

(b)

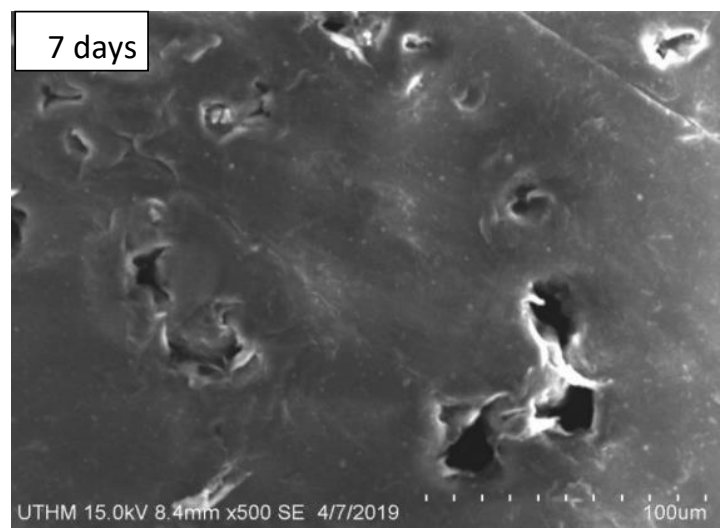

(d)

Figure 4. The surface morphology of (a) Untreated sample (b) 3 days (c) 5 days (d) 7 days.

The images from the SEM microscope only provide surface characterization for the growth of the apatite layer. To further verify and confirmation on whether the layer formed after the SBF test are apatite layer or not, X-ray Diffraction (XRD) analysis was carried out. Based on the research of [12], the XRD measurement is needed to perform at the range of 3$50^{\circ}$ in 2 theta $(\theta)$ at a $1^{\circ}$ glancing angle against the incident beam of the specimen surface.

According to previous researches, the orthorhombic phase diffraction peak for PCL is usually in the range of $20^{\circ}-25^{\circ}$ in 2 theta [6], while diffraction peak of apatite and hydroxyapatite usually occurs in the range of $20^{\circ}-40^{\circ}$ in 2 theta [12]. Although the peak of the synthetic hydroxyapatite (HA) and naturally formed apatite layer may be very similar in terms of the X-ray diffraction range, there is a significant difference in the peaks formed between these two compounds in XRD.

The X-ray diffraction (XRD) pattern of the untreated sample of PCL/PLA/PEG/n-HA is shown in Figure 5, the peak corresponds to $\mathrm{P}$ is the peak representing the main element or material in the composite which is polycaprolactone (PCL), the peak corresponds to $\mathrm{S}$ is the peak representing Starch where it is one of the key elements consist in the polymer PLA, while 
the peak labeled $\mathrm{H}$ is the peak representing synthetic hydroxyapatite that incorporated into the polymer blend [13].

The treated sample of PCL/PLA/PEG/n-HA is shown in Figure 5, the main peaks of the treated sample are similar to the untreated sample with PCL as the main material (highest peak labeled $\mathrm{P}$ ). The growth of the bone-like apatite layer can be proved by the peak labeled A, where it appears at $2 \theta=26.2^{\circ}$ and $32^{\circ}$. By comparing both XRD patterns together, it is noticeable that there are peaks of the bone-like apatite layer in the XRD pattern of the treated sample.

In general, the first thing that can be noticed by comparing both the XRD pattern is the increase in the intensity of hydroxyapatite, where the peaks of hydroxyapatite for treated samples become broader due to the growth of bone-like apatite $[14,15]$. The broadness of XRD diffraction peak depends on the crystal size and perfection or strain of that particular element. Therefore, the broader the peak, the less perfect the crystal will be [9]. The transformation of the peak of HA from the treated sample to the untreated sample (sharp HA peak to broad HA peak) is related to the microstructure of the crystal $[16,17]$.

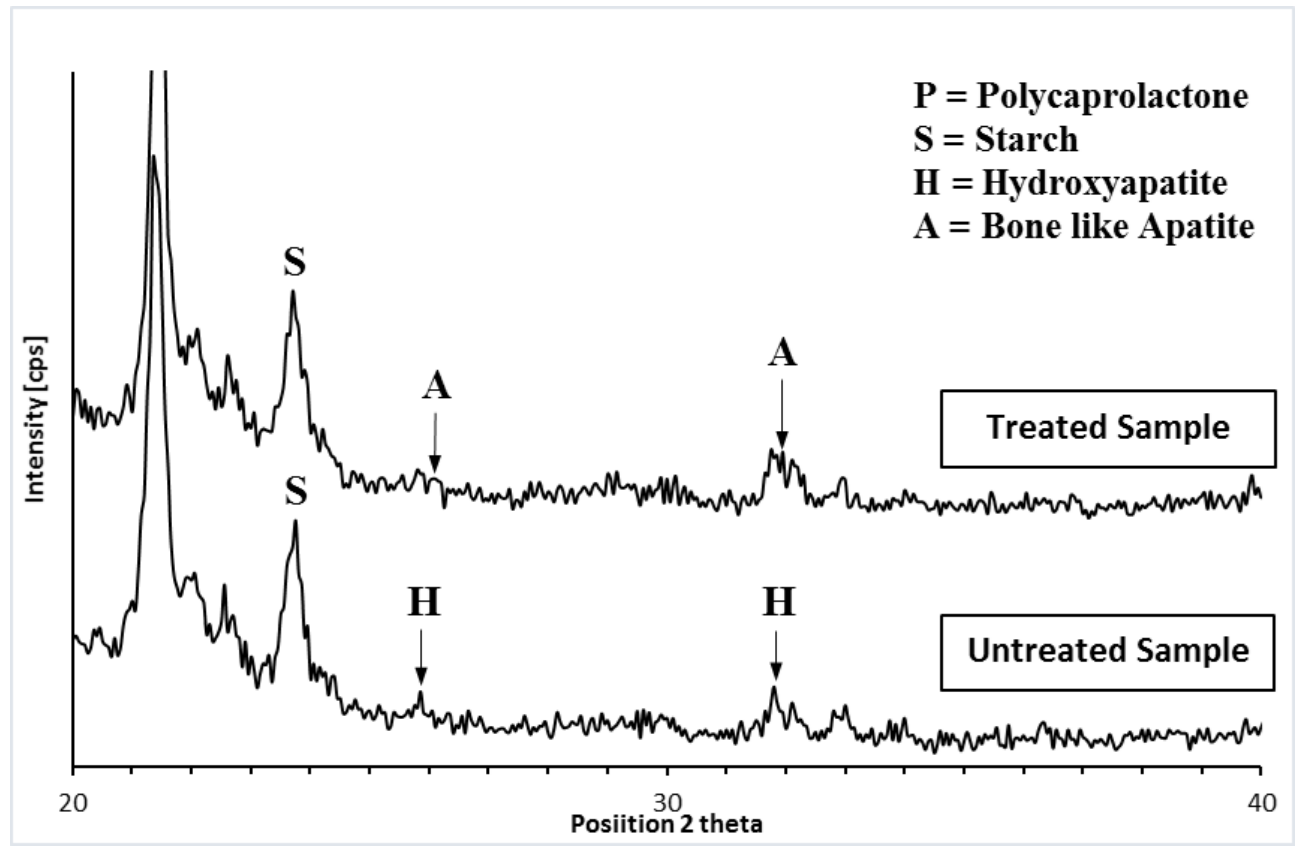

Figure 5. XRD pattern of untreated sample and treated sample.

\section{Conclusions}

PCL/PLA/PEG/n-HA polymer nanocomposites were successfully prepared. The FTIR spectra, thermal degradation, and bioactive properties of PCL/PLA/PEG/n-HA were investigated and evaluated. Thus, from this research, the following conclusions can be drawn is that the FTIR spectra of PCL/PLA/PEG/n-HA composite show that the n-HA had incorporated perfectly into the matrix of former polymer blend PCL/PLA/PEG polymer blend.

The thermal stability of PCL/PLA/PEG/n-HA composite is enhanced by the addition of HA as it was less volatile when burning by the Thermogravimetry analyzer. However, the overall performance of the composite under the thermal stability test is not as promising when compared to the thermal stability of the former PCL/PLA/PEG polymer blend. Based on the initial decomposition temperature of the PCL/PLA/PEG/n-HA composite, the composite is suitable to be used with an extruder for filament fabrication under the working temperature of below $300{ }^{\circ} \mathrm{C}$. 
Lastly, based on the morphological features of the composite under a scanning electron microscope after the samples were treated by simulated body fluid. The formation of an apatite layer on the surface of the sample shows that the incorporation of HA had revealed good bioactivity property of PCL/PLA/PEG/n-HA composite. The XRD pattern had further proved the existence of the apatite layer after immersion in simulated body fluid. Therefore, it is predicted that the PCL/PLA/PEG/n-HA has good potential for bone implant engineering.

\section{Funding}

This research received no external funding.

\section{Acknowledgments}

This research has no acknowledgment.

\section{Conflicts of Interest}

The authors declare no conflict of interest.

\section{References}

1. Kokubo, T.; Takadama, H. How useful is SBF in predicting in vivo bone bioactivity? Biomaterials 2006, 27, 2907-2915, https://doi.org/10.1016/j.biomaterials.2006.01.017.

2. Sodeifian, G.; Ghaseminejad, S.; Yousefi, A.A. preparation of polypropylene/short glass fiber composite as Fused Deposition Modeling (FDM) filament. Results in Physics 2019, 12, 205-222, https://doi.org/10.1016/j.rinp.2018.11.065.

3. Gheisari, H.; Karamian, E.; Abdellahi, M. A novel hydroxyapatite -Hardystonite nanocomposite ceramic.. Ceram. Int 2015, 4, 5967-5975, https://doi.org/10.1016/j.ceramint.2015.01.033.

4. Zhang, X.; Li, Y.; Lv, G.; Zuo, Y.; Mu, Y. Thermal and crystallization studies of nano-hydroxyapatite reinforced polyamide 66 biocomposites. Polym. Degrad. Stab 2006, 91, 1202-1207, https://doi.org/10.1016/j.polymdegradstab.2005.02.006.

5. Catauro, M.; Tranquillo, E.; Poggetto, G.D.; Naviglio, S.; Barrino, F. Antibacterial Properties of Sol-Gel Biomaterials with Different Percentages of PEG or PCL. Macromol. Symp 2020, 389, https://doi.org/10.1002/masy.201900056.

6. Abdul Haq, R.H.; Wahab, M.S.; Wahit, M.U.B. Improvement of mechanical properties of polycaprolactone (PCL) by addition of nano-montmorillonite (MMT) and hydroxyapatite (HA). Applied Mechanics and Materials 2013, 315, 815-819, https://doi.org/10.4028/www.scientific.net/AMM.315.815.

7. Yavarpanah, S.; Seyfi, J.; Davachi, S.M.; Hejazi, I.; Khonakdar, H.A. Evaluating the effect of hydroxyapatite nanoparticles on morphology, thermal stability and dynamic mechanical properties of multicomponent blend systems based on polylactic acid/Starch/Polycaprolactone. J. Vinyl Addit. Technol 2019, 25, E83-E90, https://doi.org/10.1002/vnl.21647.

8. Abdul Haq, R.H.; Marwah, O.M.F.B.; Rahman, M.N.A.; Ho, F.H.; Abdullah. H.; Ahmad, S.; Arifin, A.M.T.; Hassan, M.F.; Yunos, M.Z. Mechanical Properties Of PCL/PLA/PEG Composite Blended With Different Molecular Weight (Mw) Of Peg For Fused Deposition Modelling (Fdm) Filament Wire. International Journal of Integrated Engineering, UTHM 2018, 11, https://doi.org/10.30880/ijie.2018.10.05.028.

9. Babu, K.P.; Maruthi, Y.; Pratap, S.; Sudha, K.; Sadiku, R.; Prabhakar, M.; Song, J.; Subha, M.; Rao, K. Development and characterization of polycaprolactone (PCL)/poly ((R)-3-hydroxybutyric acid) (PHB) blend microspheres for tamoxifen drug relese studies. International Journal of Pharmacy and Pharmaceutical Sciences 2015, 7, 95-100.

10. Abdul Haq, R.H.; Rahman, M.N.A.; Arifin, A.M.T.; Hassan, M.F.; Taib, I.; Wahit, M.U.B. Thermal Properties of Polycaprolactone (PCL) Reinforced Montmorillonite (MMT) and Hydroxyapatite (HA) as an Alternate of FDM Composite Filament. Journal of Advanced Research in Fluid Mechanics and Thermal Sciences, Akademia Baru 2019, 62, 112-121.

11. Mutsuzaki, H.; Yokoyama, Y.; Ito, A.; Oyane, A. Formation of apatite coatings on an artificial ligament using a plasma- and precursor-assisted biomimetic process. Int. J. Mol. Sci 2013, 14, 19155-19168, https://doi.org/10.3390/ijms140919155.

12. Abdul Haq, R.H.; Wahab, M.S.; Wahit, M.U.B. Impact test and bioactivity properties of polycaprolactone (PCL) by addition of nano-montmorillonite (MMT) and hydroxyapatite (HA). Applied Mechanics and Materials 2013, 446-447, 1129-1133, https://doi.org/10.4028/www.scientific.net/AMM.446-447.1129. 
13. Chen, M.F.; Yang, X.J.; Liu, Y.; Zhu, S.L.; Cui, Z.D.; Man, H.C. Study on the formation of an apatite layer on NiTi shape memory alloy using a chemical treatment method. Surf. Coatings Technol 2003, 173, 229234, https://doi.org/10.1016/S0257-8972(03)00733-3.

14. Tuğba, T.A.; Memet, V.K.; Burcu, O.; Hazal, İ.; Nilhan, K.A. Bioactivated poly (vinyl alcohol) /poly(acrylic acid) based nanofiber for high-performance membrane techniques. Letters in Applied NanoBioScience 2020, 9, 819-823, https://doi.org/10.33263/LIANBS91.819823.

15. Samaneh, E.; Naser, F.; Alireza, K.A.; Sara, H. Synthesis and characterization of polymeric nanocomposites based on poly-elamineparaformaldehyde and superparamagnetic silicon dioxide loaded Iron(III) oxide coreshell composite magnetic nanoparticles. Letters in Applied NanoBioScience 2020, 9, 819-823, https://doi.org/10.33263/LIANBS91.914918.

16. Abdul Haq, R.H.; Taib, I.; Abdol Rahman, M.N.; Ho, F.H.; Abdullah, H.; Ahmad, S.; Ariffin, A.M.T.; Hassan, M.F. Mechanical Properties of PCL/PLA Composite Sample Produce from 3D Printer and Injection Molding. International Journal of Integrated Engineering 2019, 11, 102-108, https://doi.org/10.30880/ijie.2019.11.05.014.

17. Selimin, M.A.; Lee, T.C.; Haq, R.H.A.; Idris, M.I.; Abdullah, H.Z. Microstructure mechanism of the hydroxyapatite densification process. IOP Conference Series: Materials Science and Engineering 2019, 607, https://doi.org/10.1088/1757-899X/607/1/012008.

18. Bano, N.; Jikan, S.S.; Basri, H.; Adzila, S.; Badarulzaman, N, A.; Yunus, Z, M.; Asman, S. Fabrication and Properties of Polylactic Acid/Hydroxyapatite Biocomposites for Human Bone Substitute Materials. Composites in Biomedical Applications. CRC Press, 2020. 207-220. 10.1201/9780429327766-5. 